\title{
Observation on the digestive system structure and histology of Murray cod (Macculochella peelii)
}

\author{
Xiaojiang Chen, Meijian Liu, Sheng Yuan, Ziming Zhao* \\ Jiangsu Agri-animal Husbandry Vocational College, Taizhou, China \\ *Corresponding Author’s e-mail: zzm0282678@163.com
}

Keywords: Macculochella peelii, digestive system, histology.

\begin{abstract}
Murray cod (Macculochella peelii) is the latest introduced variety in China, which is large in size and is an important carnivorous freshwater fish. To provide theoretical basis for comprehensive understanding of its biological characteristics. In this study, we dissected the living body and sliced the digestive system tissue to observe the esophagus, cardiac stomach, stomach body, pyloric stomach, pyloric caeca, duodenum, foregut, hindgut, common bile duct, gallbladder, hepatopancreas, etc. We found that the digestive tract was short, the intestinal villus was dense and bifurcated. The stomach is large and Y-shaped, and there are glands in both the cardia and the stomach body, but no gland in the pyloric stomach. There are 4-5 pyloric caeca, which are thick and short. The relative gut length is less than 1 . The structure of digestive system shows the characteristics of adapting to its carnivorous habit.

Macculochella peelii is a freshwater fish mainly distributed in Australia and South America. It is a famous freshwater culture variety in Australia. Because of its special appearance and delicious taste, it is called "national treasure fish". It is one of the largest freshwater fish in the world. Its growth speed is fast, and its ability to adapt to the environment is strong. Moreover, it is not only suitable to be cultured as an edible fish, but also suitable for viewing. Because of its delicate meat, no fishbone between muscles, small visceral mass and high meat content, it has high commodity value. The fish has high requirements for water quality and it has certain requirements for illuminance, and the feed conversion rate is high, so the fish is suitable for industrialized culture. In 1999, it was first introduced to Taiwan from Australia, and in 2001, it was introduced to the mainland of China. At present, as a new breed of freshwater culture in China, the fish has attracted much attention and has great prospects for development. There are many reports on the study of haddock [1-6]. Through this paper, we observe the digestive system and histology of Macculochella peelii, and provide theoretical basis for comprehensive understanding of its biological characteristics, more effective production, processing and domesticated feeding of artificial compound feed, and further expansion of its breeding production scale.
\end{abstract}

\section{Materials and methods}

The Macculochella peelii was taken from Shandong Qingdao qihao nutrition technology co. LTD. First, culture it in water for 3-5 days, then measure its body length and body weight, and then dissect and observe it, and then weigh its intestinal weight, liver weight and visceral weight, and then take the typical tissues of its digestive system, soak it in with $4 \%$ formalin, and fix it with Bouin solution, and use paraffin-cut section method to cut it according to the conventional histological method. The slice thickness is $3-4 \mu \mathrm{m}$, and then carry out HE staining, use Motic microscope to observe it, and then measure it, take photos and mark it. Digestive tract index ${ }^{[7]}$ : relative gut length (length of intestine / length of body), intestine weight index (weight of intestine / weight of body), Hepato-somatic Index (weight of liver / weight of body) and viscera weight index (weight of viscera / weight of body). 


\section{Observation of digestive system tissue}

\subsection{Digestive tract}

The digestive system of Macculochella peelii is relatively simple. It is composed of mouth, pharynx, esophagus, cardiac stomach, stomach body, pyloric stomach, pyloric caeca, intestine, anus, hepatopancreas, gallbladder, etc. Digestive tract index of Macculochella peelii(Table 1)

Table 1. Digestive tract index of Macculochella peelii

\begin{tabular}{ccccccc}
\hline & $\begin{array}{c}\text { Body } \\
\text { length } \\
(\mathrm{cm})\end{array}$ & $\begin{array}{c}\text { Body } \\
\text { weight } \\
(\mathrm{g})\end{array}$ & $\begin{array}{c}\text { Relative } \\
\text { gut length }\end{array}$ & $\begin{array}{c}\text { Intestine } \\
\text { weight } \\
\text { index }\end{array}$ & $\begin{array}{c}\text { Hepato-somatic } \\
\text { Index }\end{array}$ & $\begin{array}{c}\text { Viscera weight } \\
\text { index }\end{array}$ \\
\hline Maximum & 40.1 & 1585.8 & 0.596 & 0.0055 & 0.0166 & 0.065 \\
Minimum & 33.2 & 945.5 & 0.532 & 0.0043 & 0.0146 & 0.054 \\
Average & 37.3 & 1296.9 & 0.556 & 0.0048 & 0.0158 & 0.057 \\
& \pm 2.86 & \pm 261.9 & \pm 0.028 & \pm 0.0005 & \pm 0.007 & \pm 0.005 \\
\hline
\end{tabular}

2.1.1 Mouth and pharynx

The maxilla and submaxillary of Macculochella peelii are wide, with large mouth crack. The maxilla is slightly longer than the submaxillary. In both the maxilla and submaxillary, there are sharp teeth of the same size. There are 3 rows of teeth in the maxilla, and there are palatal teeth and vomer teeth (Fig. 1). There are 1 row of teeth in the submaxillary (Fig. 2). As for the pharynx, there is also a tooth belt distributed at the base of the branchial arch bone.

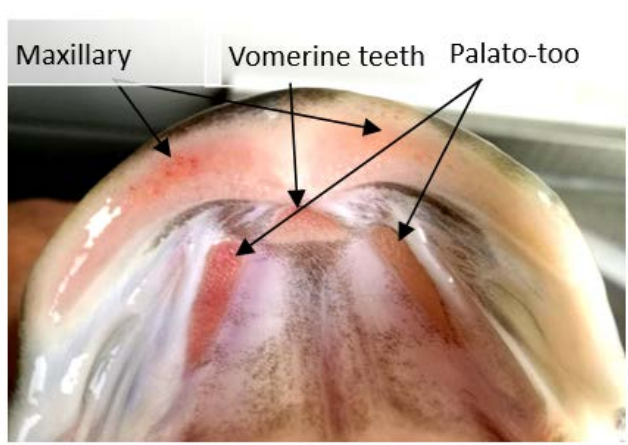

Fig 1. Maxillary

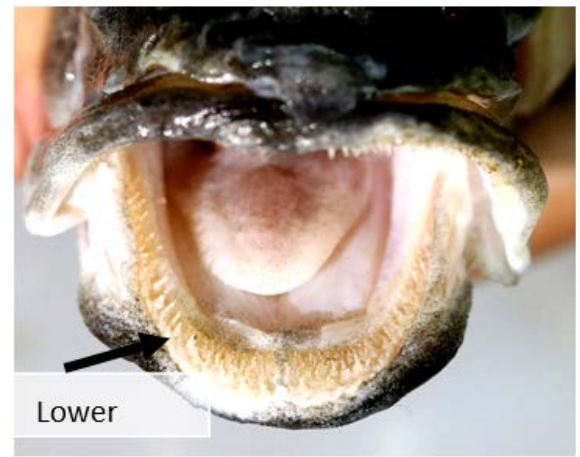

Fig 2. Submaxillary

\subsubsection{Esophagus}

The esophagus is short and there are 19-21 wrinkles. The posterior part of the esophagus is connected with the stomach, which is divided into four layers: mucosa, submucosa, muscle layer and serosa. The mucosa wrinkles in the direction towards the cavity. On the surface of the mucosa, there are multiple-layer flat epithelium covering it. Among the epithelial cells, there are densely distributed goblet cells (Fig 4). On the top layer, there are aging epithelium. The distribution of connective tissue in submucosa is much and thick.

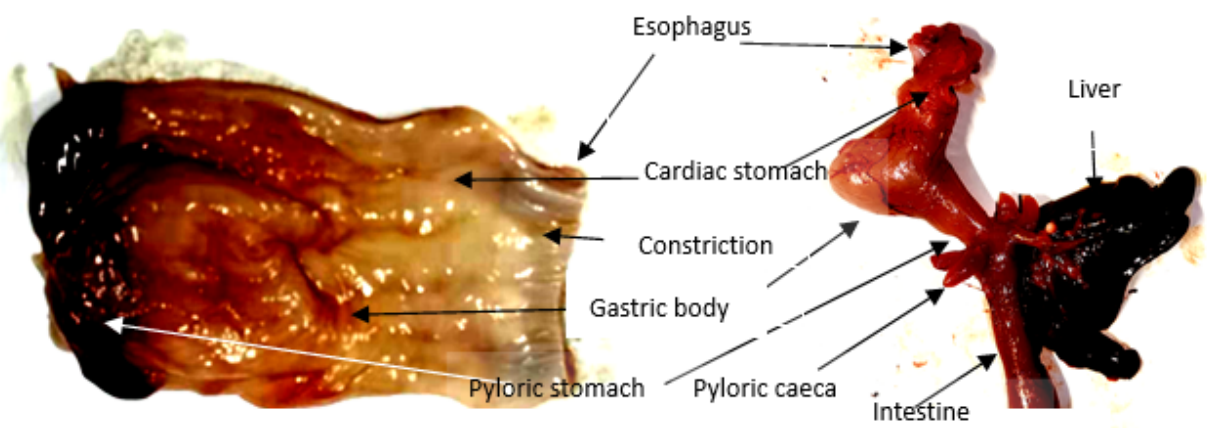

Fig. 3. Digestive tract and digestive gland of Macculochella peelii 


\subsubsection{Cardiac stomach}

It is located at the back of the esophagus. There are obvious constrictions between esophagus and cardiac stomach. The surface of the cardiac stomach is relatively smooth, and the wrinkles are not obvious (Fig 3). In the interior of the cardiac stomach, there are many wrinkles arranged compactly and longitudinally, and on them, there are many secondary wrinkles, the top of which is flat on the whole, but there are much gastric pits. With regard to the Centrally distributed gastric glands in the cardiac stomach, most of the glands are like branch tubes and they penetrate into the connective tissue, and open in the gastric pits (Fig 5). Moreover, the arrangement of gastric gland cells is tight and the blood vessels are rich. Its muscle layer is thick.

\subsubsection{Gastric body}

The gastric body is in the shape of sacs and the wrinkles are large. Slice the tissue, and then observe it that there are several gastric glands in it, the number of which is denser than the glands in the cardiac stomach, and it is tubular. There are more and deeper wrinkles on the surface than on the cardiac part. The epithelium of the mucosa consists of a single layer of closely arranged columnar epithelium. The epithelium sinks to form a gastric pit. The side of the secondary wrinkle is a surface mucus cell. The muscle layer of the stomach body is thicker than that of the cardiac part (Fig. 6).

\subsubsection{Pyloric stomach}

The boundary between the pyloric part and the stomach body is obvious. There are many wrinkles forming in the mucosa, and the basement layer is relatively thick. There are few wrinkles on the inner surface of the pyloric stomach (Fig 3). The mucosa epithelium is a single-layer columnar epithelium with no gastric glands distributed on it (Fig. 7).

\subsubsection{Pyloric caeca}

There are 4 or 5 pyloric caeca (Fig 3). The sizes and lengths of the pyloric caeca of the individuals with 4 pyloric caeca are basically the same, while for the individuals with 5 pyloric caeca, the sizes and lengths of 4 pyloric caeca are basically the same, the other one is small and short, and its length is about $1 / 3$ of the average length of the other four. In individuals with four or five pyloric caeca, the common bile duct will open at the base of the pyloric caeca that is relatively larger.

The structure of pyloric caeca is similar to that of intestine. The outermost layer of the muscle layer is the outer circular muscle, and the inner layer is the inner circular muscle. The mucosa villus protrudes inward and form branches, which are crisscross and reticulate. There are a few goblet cells in the columnar epithelial cells of villi (Fig. 8).

\subsubsection{Duodenum}

It is located in the back of the stomach, and its wall is thin. The outermost layer is the outer circular muscle and the inner layer is the inner circular muscle. Many wrinkles protrude from the basement and the mucosa upper layer of mucosa to the interior, and the mucosa epithelium is a single-layer columnar epithelium. The basal layer is thicker than the pyloric caeca, without bifurcations, and there are a small number of goblet cells distributed (Fig 9).

\subsubsection{Midgut}

It connects the duodenum and rectum, and its basic structure is the same as that of the general intestine. The outermost layer is composed of several oval single-layer flat epithelial cells. The outer circular muscle is thin and clings to the single-layer flat epithelial cells. The inner circular muscle is thicker than the outer circular muscle. The number of goblet cells on chorion increases from front to back (Fig 10). 


\subsubsection{Rectum}

The outermost layer is composed of several oval single-layer flat epithelial cells. The outer circular muscle is thin and clings to the single-layer flat epithelial cells. The inner circular muscle is thicker than the outer circular muscle. The number of goblet cells on the villi is large and there are many hidden hollows (Fig 11).

\subsection{Digestive gland}

\subsubsection{Hepatopancreas}

It is located in the front of the abdominal cavity of the fish. Two kinds of tissues, liver and pancreas, are embedded together. At the same time, in the pyloric caeca, duodenum and mesentery near the common bile duct, it can be found that there are seven large pancreatic islands, six of which are round and one is long oval. Tissue sections show that there are many pancreases embedded in the liver, and the hepatic cord cells are large, the nucleus is spherical or oval, and there are abundant blood vessels in the liver, and the boundary between the liver and pancreas is clear (Fig 12).

\subsubsection{Common bile duct}

The common bile duct is thick and opens at the base of one of the pyloric caeca. The muscle layer of the common bile duct is relatively thick, and the outside is surrounded by a single-layer flat cell. In the cavity, there are villus, which are composed of a layer of columnar epithelial cells, and there are goblet cells spaced between them (Fig 13).

\subsubsection{Gall bladder}

The gallbladder is located under the liver and is oval. The outer part of the gallbladder is composed of a single-layer of flat epithelium. The columnar cell nucleus in the cavity are large and orderly arranged. The intercellular space is clear (Fig 14).

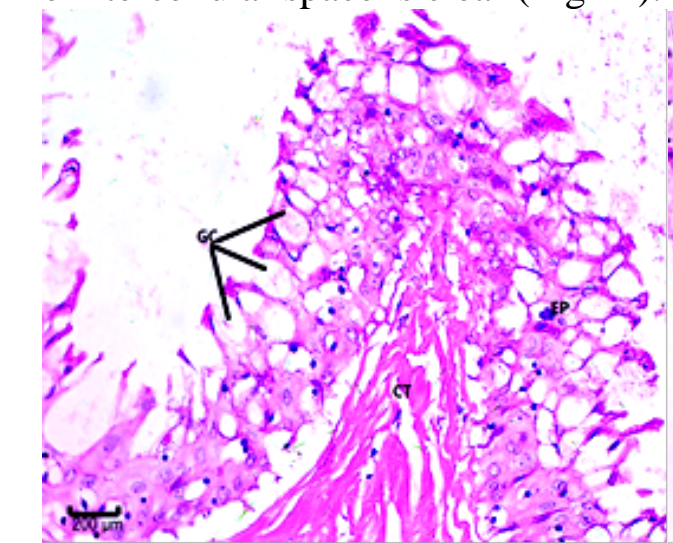

Fig 4. Esophagus

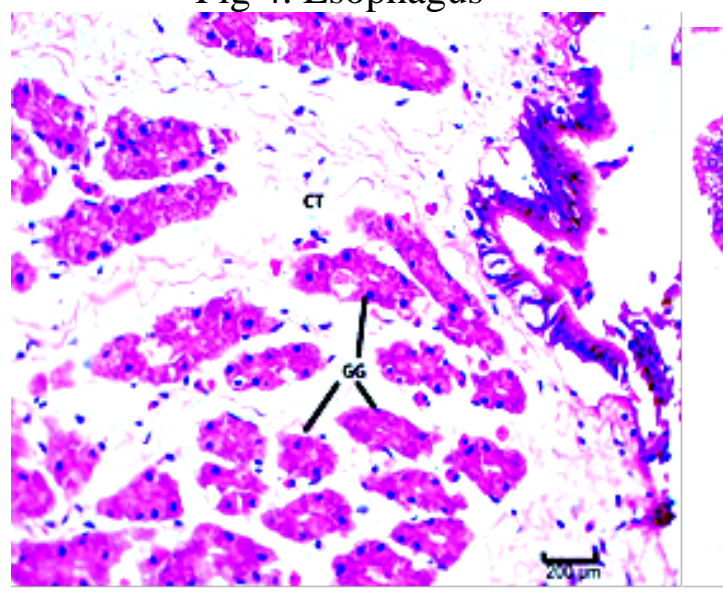

Fig 6. Gastric body

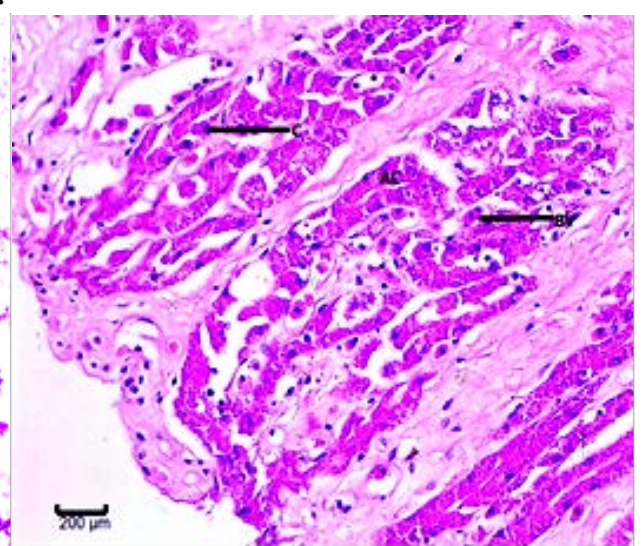

Fig 5. Cardiac stomach

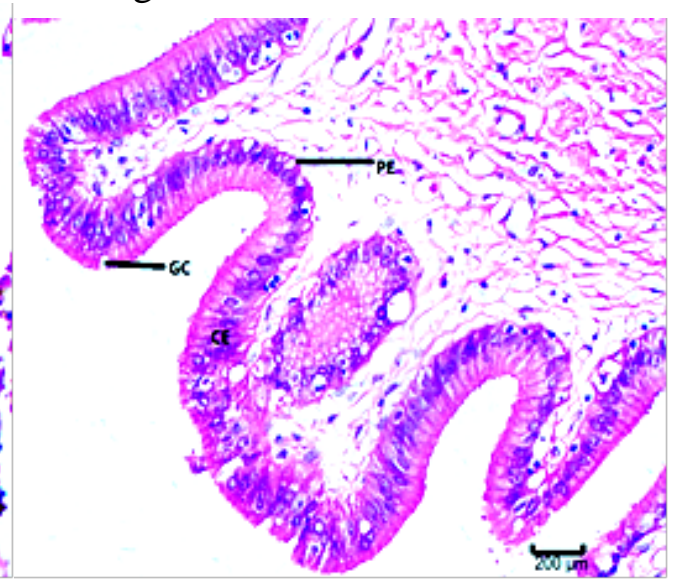

Fig 7. Pyloric stomach 


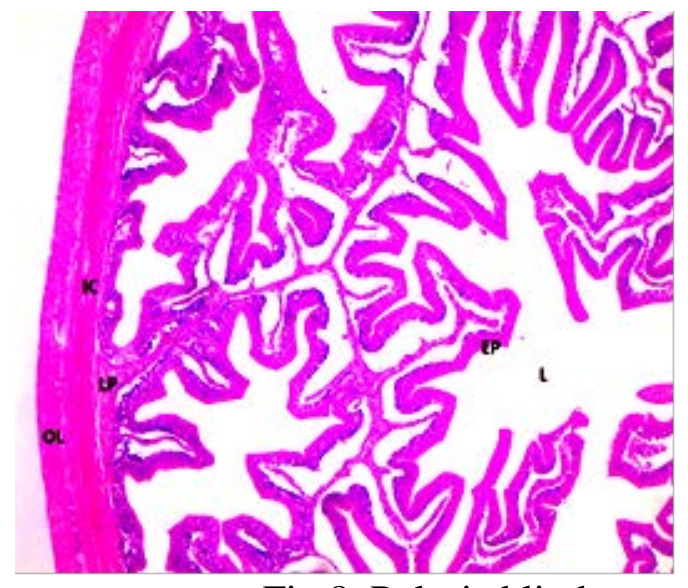

Fig 8. Pyloric blind sac

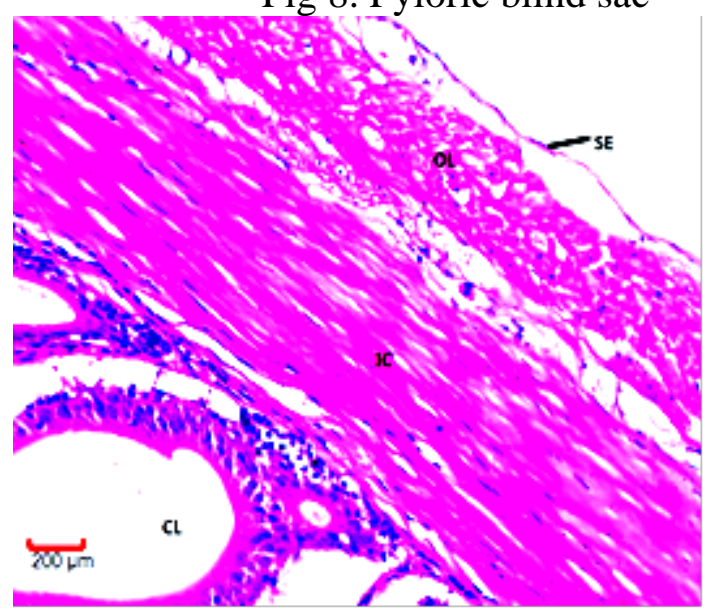

Fig 10. Midgut

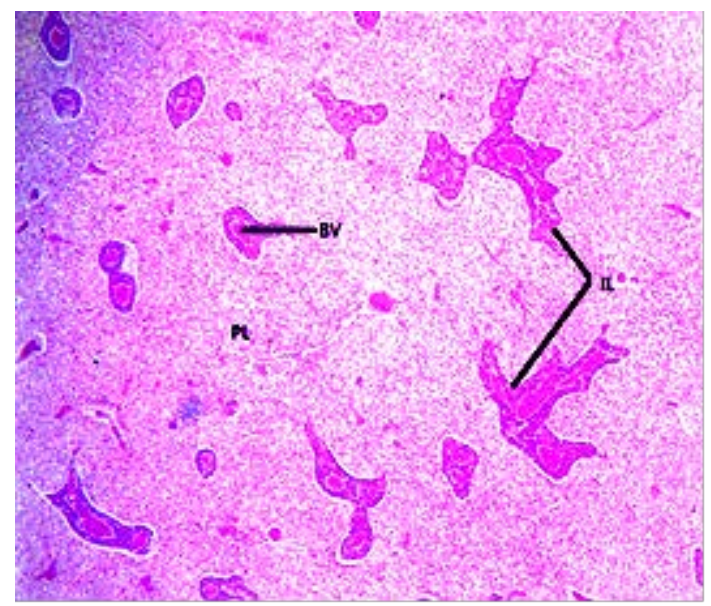

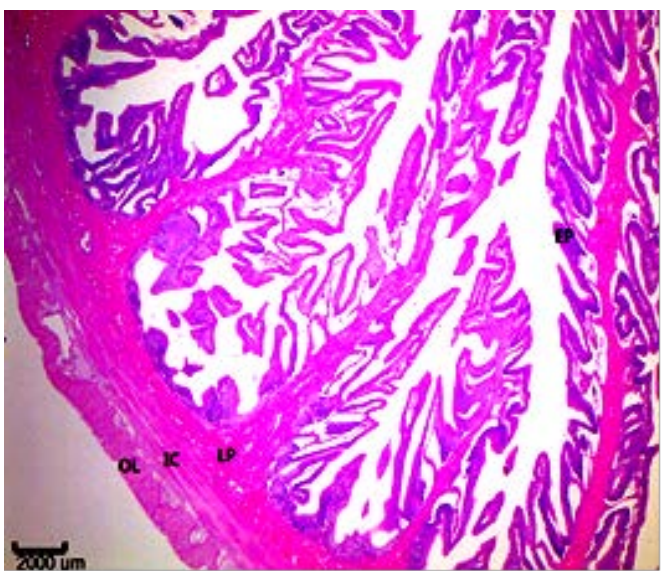

Fig 9. Duodenum

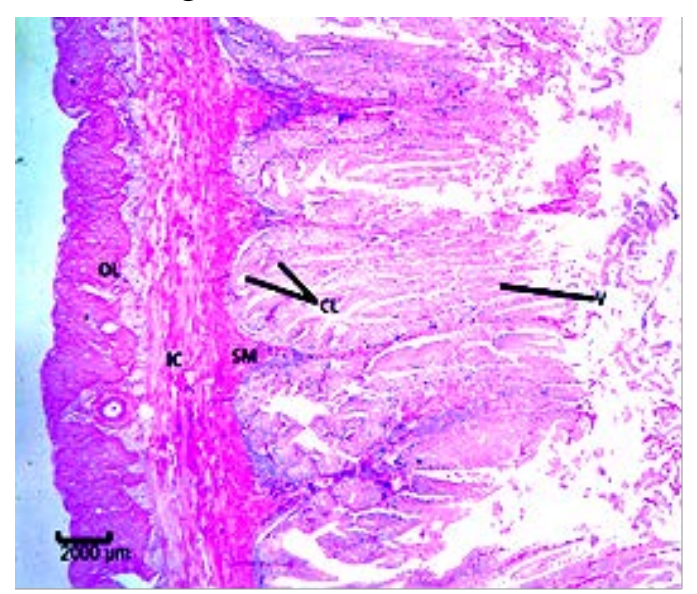

Fig 11. Rectum

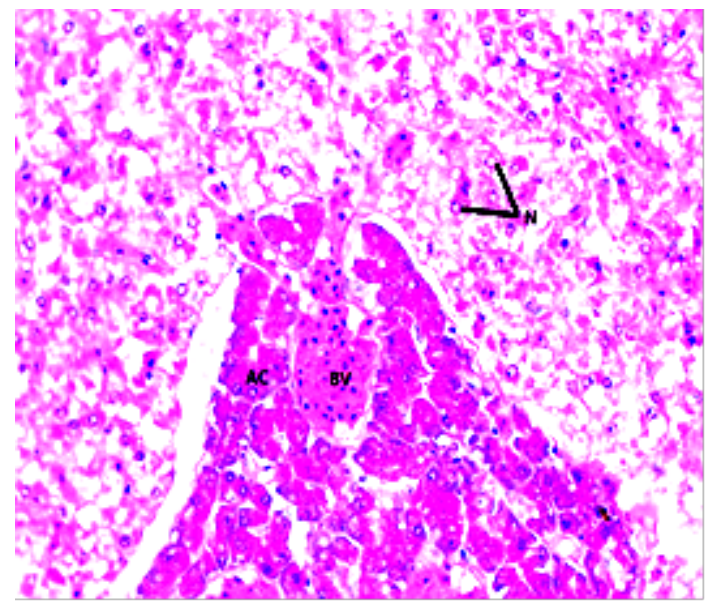

Fig 12. Hepatopancreas 


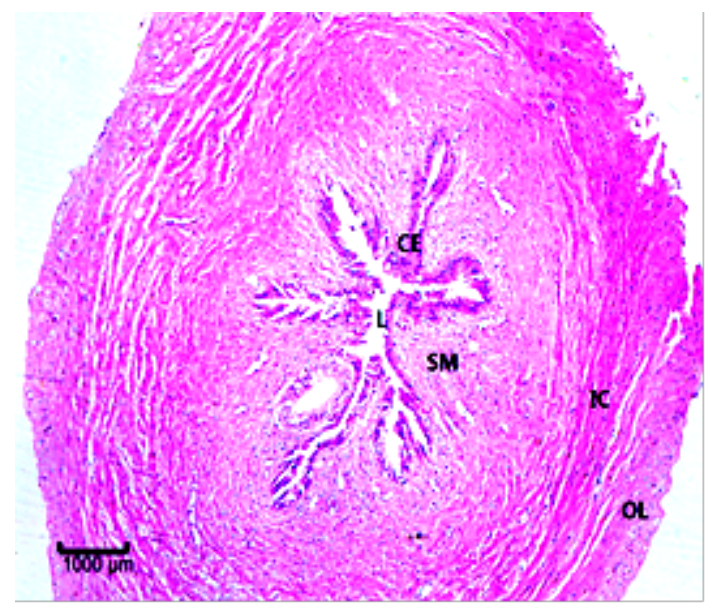

Fig 13. Common bile duct

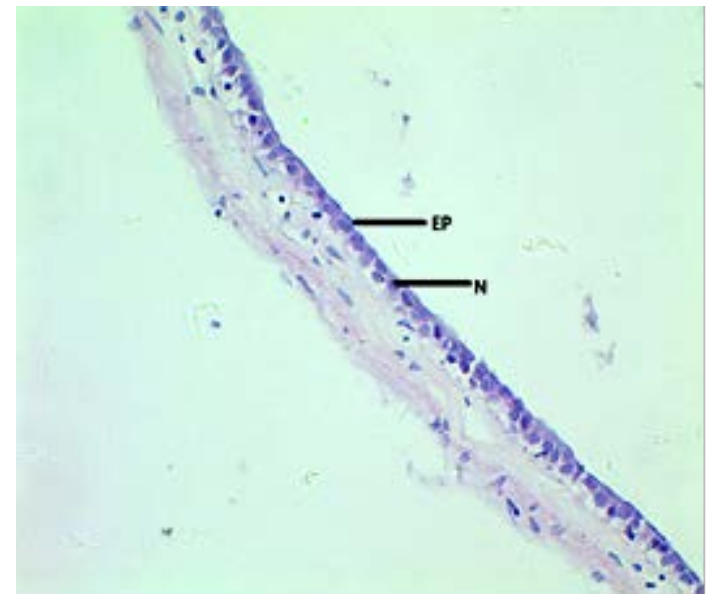

Fig 14. Gall bladder

Note: OC: outer longitudinal muscle; IC: inner circular muscle; SM: basal layer; CL: hidden hollows; V: intestinal villi; BV: blood vessel; PC: hepatic cord cells; IL: pancreas; N: nucleus; AC: pancreatic duct; CE: villi; L: cavity; SE: single-layer flat epithelial cell; C: gland cell; CT: connective tissue; EP: multi-layer flat epithelial cell; GC: goblet cell; GG: fundic gland; PE: submuscular membrane

\section{Discussion}

\subsection{Digestive tract index and feeding habits of Macculochella peelii}

Through the digestive system, fish can absorb and digest food and nutrients, so as to further obtain the nutrients and energy needed for a series of life processes such as growth, development and reproduction. According to the different feeding ways and food types, fish are generally divided into phytophagous fish, omnivorous fish and carnivorous fish. Because of the different characteristics of the digested food, there are great differences in the shape and structure of the digestive system of the fish with different feeding habits. Therefore, digestive tract index, such as relative gut length ${ }^{[8]}$, intestine weight index, Hepato-somatic Index and visceral weight index, can directly reflect the feeding habits. The length of intestines of general carnivorous fish is 1/3 1/4 of body length; that of herbivorous fish is more than 2 5 times of body length; that of omnivorous fish is between the two above. Because of the stomach, carnivorous fish can hold more food, and the food can stay for a longer time, which is conducive to digestion, and its relative gut length is small, generally less than 1; for omnivorous and herbivorous fish, because there is no stomach, its relative gut length is large, and most of which are greater than 1, for example, the relative gut length of Ctenopharyngodon idealus and Hypophthalmichthys molitrix are 2 8, and the relative gut length of Aristichthys nobilis is about 4.58 , and the structures of digestive systems of other fishes also prove this phenomenon ${ }^{[9-13]}$. The visceral mass of carnivorous fish is smaller, and its visceral weight index is smaller than that of omnivorous or herbivorous fish, and the visceral weight index of Macculochella peelii is 0.058, which is similar to that of Protosalanx hyalocranius (visceral weight index is 0.038$)^{[14]}$, but smaller than that of Siganus canaliculatus (visceral weight index is 0.157$)^{[15]}$. The liver is the largest digestive gland of fish. The bile secreted by the liver and the pancreatic juice secreted by the pancreas can be used to decompose animal food. The Hepato-somatic Index of carnivorous fish is smaller than that of omnivorous or phytophagous fish ${ }^{[16,17]}$. Macculochella peelii has a thick and developed Y-shaped stomach ${ }^{[18]}$, accounting for $16.5 \%$ of the whole digestive tract. It has obvious cardiac part, pyloric part and blind sac. The intestine is relatively thick and only shows two bends in the abdominal cavity. The relative gut length is less than 1 , the average visceral weight index is 0.057 and the average Hepato-somatic Index is 0.0158. The digestive tract index reflects the digestive tract characteristics of carnivorous fish. 


\subsection{The relationship between the histological and structural characteristics of digestive system and feeding habit}

The innermost layer of the digestive tract tissue structure of Macculochella peelii is chorion, and the digestive tract chorion is more than that of other fishes. Outside the chorion is the epithelial layer, which is composed of a single-layer epithelial cells, and the nucleus is relatively large, and the boundary between cells is not clear, and a thin membrane is wrapped outside the epithelial layer. At the oesophagus of Macculochella peelii, there are broad, short and flat mucosal wrinkles, so when Macculochella peelii swallows food, the esophagus can be fully expanded, so that the food can pass through the oesophagus smoothly, reducing the stay time of food in the oesophagus, so as to prevent food from damaging the surface of the digestive tract. The stomach of Macculochella peelii is relatively developed, and the differentiation of each part is obvious. The stomach wall is very thick, and the stomach glands are many and developed, which makes the mucosa significantly thicker than other parts of the digestive tract, and this characteristic is similar to that of Chromileptes altivelis ${ }^{\text {[19] }}$. Macculochella peelii is an integration of liver and pancreas, while the pancreatic islands are round or oval, scattered on the mesentery. Although the two tissues of liver and pancreas are mixed together, they are still two independent organs, and secretions are transported by their own ducts, which is similar to that of Cyprinidae. The developed stomach and stomach gland, mucosa wrinkles and pyloric sphincter of Macculochella peelii can enlarge the stomach capacity and ingest more food, and then the food is digested under the action of pepsinogen and hydrochloric acid secreted by the stomach gland, which also conforms to the characteristics of carnivorous fish. The number of pyloric caeca (only 4 5) in Macculochella peelii is less than that in Chromileptes altivelis (10 13). The structure characteristics of pyloric caeca is similar to the intestine, which can increase the absorption area of the intestine. The secretion has strong digestive enzyme activity, which can make the food fully digested and absorbed in the intestine ${ }^{[20]}$. The intestinal epithelium of Macculochella peelii folds repeatedly, forming rich wrinkles. In the hindgut, the circular muscle layer is more developed than that of the foregut, which also reflects the perfection of digestive system function.

\section{Acknowledgments}

This research was financially supported by Jiangsu Three New Engineering's (Grant No. Y2017-42). It was also supported by “Qing Lan Project of Jiangsu Province” (Teacher Jiangsu (2018) 12) and "311 Talents Project in Taizhou" (2017Ш-804).

Conflict of Interest: The authors declare that they have no conflict of interest.

Ethical approval: “All applicable international, national, and/or institutional guidelines for the care and use of animals were followed by the authors.”

\section{References}

[1] WENG B Q, LUO T Y, LIU Y, et al. Biological Characteristics,Propagation and Aquaculture of Murray Cod (Maccullochellapeelii) [J]. Fujian Journal of Agricultural Sciences,2016,31(01):89-94.

[2] LIU J L, IV J. study on protease activity in digestive tract of silver perch from Australia [J]. Journal of Hydroecology,2007(05):9-11.

[3] CHEN Y l, ZHU X P, LIU Y H. Australian freshwater cod and artificial breeding [J]. Freshwater Fisheries, 1999 (7): 6-7.

[4] Turchini G , Gunasekera R M , De Silva S . Effect of crude oil extracts from trout offal as a replacement for fish oil in the diets of the Australian native fish Murray cod Maccullochella peelii peelii[J]. Aquaculture Research, 2015, 34(9):697-708.

[5] Gooley, GJ, Anderson, TA, Appleford, P. Aspects of the reproductive cycle and gonadal development of Murray cod, Maccullochella peelii peelii (Mitchell) (Percicthidae), in Lake Charlegrark and adjacent farm ponds, Victoria, Australia[J]. 1995, 46(4):723-728. 
[6] Turchini G M, Francis D S, De Silva S S. Fatty acid metabolism in the freshwater fish Murray cod (Maccullochella peelii peelii) deduced by the whole-body fatty acid balance method. [J]. 2006, 144(1):110-118.

[7] AN L, MENG Q L, LI X, et al. Study on digestive tract index and main digestive enzyme activity of Murray cod [J]. Journal of Yangtze University (Natural Science Edition), 2017(14):32 36.

[8] LIN H R. Fish physiology [M]. Guangdong Higher Education Press, 2011.

[9] PAN Q S, GUO G Q, FANG Z P, et al. The comparative anatomy studies on digestive system of 6 fish species of stomach-containing teleost in freshwater [J]. Journal of Huazhong Agricultural University, 1996(5):463-469.

[10] BI B, SUN Z W, MAO T Q, et al. Relationship between digestive tract structure and feeding habits in common carp, grass carp, silver carp and bighead carp [J]. Chinese journal of fisheries, 2011(01):28-31.

[11] Lin Haoran. Digestive tract of five species of Cyprinidae [J] Journal of Zhongshan University (Natural Science Edition). 1962(03):67-80.

[12] ZENG D, YE Y T. Study on the food habit and digestive system structure of fish [J]. Journal of Southwest Agricultural University, 1998(4):361-364.

[13] QIAN Y X, YANG W G, LU K H. Studies on the Digestive Tract Index and the Digestive Enzyme of the Large Icefish Protosalanx hyalocranius [J]. Reservoir fisheries, 2001(05):8-9.

[14] YANG J H, ZHANG L Z, ZHUANG P, et al. The digestive tube index and the activity distribution of three kinds of digestive enzymes in the digestive organs of cultured Siganus canaliculatus [J]. Marine sciences, 2009, 33(7):43-50.

[15] XIONG H L. Study on Anatomy Structure of the Digestive System of Coreoperca whiteheadi [J]. Journal of Anhui Agricultural Sciences 2011, 39(7):4015-4017.

[16] Wang Y N. Comparative study on digestive system of four freshwater economic fishes [D]. Hunan Agricultural University, 2008

[17] AN L, DONG X S, ZHANG Y H, et al. Study on the shape characteristics and the structure of internal digestive system of Murray cod [J]. Journal of Yangtze University (Natural Science Edition), 2013(6):29-32.

[18] OU Y J, GOU X W, LI J E. Histological and histochemical studies on digestive system of Cromileptes altivelis [J]. Marine Fisheries, 2011,33(03):289-296.

[19] YE M, WU C Y, WANG Q, et al. Rough extraction and some properties of proteinase from digestive tube of eel [J]. Acta Oceanologica Sinica 2000,22(3):132-136.

[20] YANG X P, ZHANG J Z, ZENG K W, et al. Variations of Activities of Protein Enzymes in the Mandarin Fish Siniperca chuatsi under Different Feeding Conditions [J]. Journal of Hydroecology, 2003, 23(5):11-14. 